PROCEEDINGS OF THE

AMERICAN MATHEMATICAL SOCIETY

Volume 137, Number 11, November 2009, Pages 3823-3832

S 0002-9939(09)09957-2

Article electronically published on July 14, 2009

\title{
REITER NETS FOR SEMIDIRECT PRODUCTS OF AMENABLE GROUPS AND SEMIGROUPS
}

\author{
BENJAMIN WILLSON
}

(Communicated by Nigel J. Kalton)

\begin{abstract}
In this paper we study Reiter nets for semidirect products of locally compact groups. A Reiter net is a net in $L^{1}(G)_{1}^{+}$which satisfies Reiter's condition (P1). These are nets of means which converge to left invariance in norm uniformly on compact subsets of $G$. We provide two methods to combine Reiter nets for two groups to create a Reiter net for their semidirect product. We also present analogous results for combining Følner nets for locally compact groups and for Reiter nets for semidirect products of discrete semigroups.
\end{abstract}

\section{INTRODUCTION}

A locally compact group $G$ is said to be amenable if there exists $m \in\left(L^{\infty}(G)^{*}\right)_{1}^{+}$ (the set of positive linear functionals of norm one on $L^{\infty}(G)$ ) which is invariant under left translation. In [7, Hulanicki showed that Reiter's condition, introduced in [17, is equivalent to amenability. More specifically, he showed that $G$ is amenable if and only if there is a net $\left(f_{\alpha}\right)_{\alpha}$ in $L^{1}(G)_{1}^{+}$such that $\left\|l_{x}^{*} f_{\alpha}-f_{\alpha}\right\|$ tends to zero uniformly in $x$ on compact subsets of $G$. Throughout this paper we will refer to such nets as Reiter nets.

In [3, Følner gave a necessary and sufficient condition for a discrete group to be amenable. Namioka [15] extended this research and demonstrated the connection between sets which arise in the Følner condition and Reiter nets. Further connections have been drawn between Reiter nets, Følner nets and approximate diagonals of the group algebra $L^{1}(G)$ by Stokke in [19] and 20. Additionally, Reiter nets have been used to construct bounded approximate identities and virtual diagonals of the Fourier algebra $A(G)$ (see [14, [10, and [18]).

In this paper we shall present two methods for constructing Reiter nets for semidirect products of locally compact groups. Both methods provide a Reiter net for a semidirect product based upon Reiter nets for the original two groups. These results can be applied to construct Følner nets for semidirect products providing new insights on a result of Janzen [8].

Additionally, similar results are shown for semidirect products of discrete semigroups. In the discrete case, Reiter nets are equivalently characterized as strongly converging to left invariance (pointwise). Such nets play an important role in the

Received by the editors December 16, 2008, and, in revised form, March 3, 2009.

2000 Mathematics Subject Classification. Primary 43A07, 22D05.

Key words and phrases. Reiter nets, semidirect product, strongly regular nets, Følner nets, amenable groups, semigroups.

(C)2009 American Mathematical Society Reverts to public domain 28 years from publication 
approximation of fixed points for semigroups of non-expansive mappings (see [1], 12. where they are referred to as strongly left-regular nets). Recently, Hindman and Strauss [6] have shown that for a left-cancellative left-amenable semigroup, $S$, the weak*-closed convex hull of the left-invariant means arising from Følner nets contains all the left-invariant means on $S$. (If $S$ is a group, then this is a result of Chou [1].)

This paper is composed of results which were motivated by work done in the author's M.Sc. thesis [21, written under the supervision of Professor Anthony T.-M. Lau at the University of Alberta. The author wishes to thank Professor Lau for the motivation and suggestions he provided. The author is also grateful to R. Stokke for his many useful comments and to the referee for many valuable suggestions. The financial support of NSERC and the University of Alberta is gratefully acknowledged.

\section{Preliminaries}

Let $G$ be a locally compact group with fixed left Haar measure $\lambda_{G}$.

Definition 2.1. We use $L^{\infty}(G)$ to denote the Banach space of complex-valued essentially bounded functions on $G$ with respect to $\lambda_{G}$ and equipped with the essential supremum norm. A functional in the dual space of $L^{\infty}(G), m \in L^{\infty}(G)^{*}$, is called a mean on $L^{\infty}(G)$ if

$$
\|m\|=1=m\left(\chi_{G}\right)
$$

where $\chi_{G}$ is the constant function which is defined to be 1 at every point in $G$. A mean $m$ is left invariant if for any $\phi \in L^{\infty}(G)$ we have $m(\phi)=m\left(l_{g} \phi\right)$ for any $g \in G$, where $l_{g} \phi(x)=\phi(g x)$. G is (left) amenable if there exists a left-invariant mean on $L^{\infty}(G)$.

The class of amenable locally compact groups includes all abelian and compact groups. However, the free group on two generators, $\mathbb{F}_{2}$, or any locally compact group containing $\mathbb{F}_{2}$ as a closed subgroup is not amenable.

Definition 2.2. We define a left action of $G$ on elements of $L^{1}(G)$, which we shall call left translation, by:

for all $g, x \in G$ and $f \in L^{1}(G)$.

$$
\left(l_{g}^{*} f\right)(x)=f\left(g^{-1} x\right)
$$

Let $\left(f_{\alpha}\right)_{\alpha}$ be a net in $L^{1}(G)_{1}^{+}$(the elements of $L^{1}(G)$ which are positive and of norm one). We say that $\left(f_{\alpha}\right)_{\alpha}$ is a Reiter net if $\left\|l_{x}^{*} f_{\alpha}-f_{\alpha}\right\|_{L^{1}(G)} \rightarrow 0$ uniformly in $x$ on compact subsets of $G$. That is, for every $K \subset G$ compact and every $\varepsilon>0$ there exists $\alpha_{0}$ such that for all $\alpha>\alpha_{0}$ and all $x \in K$,

$$
\left\|l_{x}^{*} f_{\alpha}-f_{\alpha}\right\|_{L^{1}(G)}<\varepsilon \text {. }
$$

In [17, Reiter showed that every locally compact abelian group admits a Reiter net and used this property to prove several interesting results. Hulanicki [7] then showed that the class of locally compact groups which admit Reiter nets is precisely the class of amenable groups.

Definition 2.3. Let $N$ and $H$ be locally compact groups with $H$ acting on $N$; i.e., there exists $\tau$, a group homomorphism from $H$ to $\operatorname{Aut}(N)$ such that $(n, h) \rightarrow \tau_{h}(n)$ is continuous with respect to the product topology on $N \times H$, where $\operatorname{Aut}(N)$ is the group of continuous group automorphisms of $N$. We say that $G:=N \rtimes_{\tau} H$ is 
the semidirect product of $N$ and $H$ with respect to $\tau$ if $G$ is the group consisting of elements of the form $(n, h)$, where $n \in N$ and $h \in H$, equipped with multiplication given by:

$$
\left(n_{1}, h_{1}\right) *\left(n_{2}, h_{2}\right)=\left(n_{1} \tau_{h_{1}}\left(n_{2}\right), h_{1} h_{2}\right) .
$$

If $G$ is equipped with the product topology, then $G$ is a locally compact group. We will refer to $N$ and $H$ as the factor groups of $N \rtimes_{\tau} H$.

If $f: N \rightarrow \mathbb{C}$ and $g: H \rightarrow \mathbb{C}$, then we define $f \times g: N \rtimes_{\tau} H \rightarrow \mathbb{C}$ via $f \times g(n, h)=f(n) g(h)$.

It is well-known (cf. [5, 15.29]) that the left Haar measure of a semidirect product depends on the left Haar measures of the factor groups. It can be defined in the following way:

$$
d \lambda_{N \rtimes_{\tau} H}(n, h)=\delta(h) d \lambda_{N}(n) d \lambda_{H}(h),
$$

where $\delta(h)=\frac{\lambda_{N}(A)}{\lambda_{N}\left(\tau_{h}(A)\right)}$ for each measurable $A \subset N$. Then $\delta$ is a continuous group homomorphism from $H$ to $\mathbb{R}^{+}$.

From the action, $\tau$, of $H$ on $N$ we define

$$
\begin{aligned}
T: H & \rightarrow B\left(L^{1}(N)\right) \\
& h \mapsto T_{h}
\end{aligned}
$$

via $\left(T_{h} f\right)(n)=f\left(\tau_{h^{-1}}(n)\right) \delta(h)$ for $f \in L^{1}(N), n \in N, h \in H$. It can be easily checked that each $T_{h}$ is a linear isometry and preserves convolution.

\section{REITER NETS IN $L^{1}\left(N \rtimes_{\tau} H\right)_{1}^{+}$}

When investigating Reiter nets for semidirect products, it is natural to wonder how Reiter nets for $N$ and $H$ may be combined to produce a Reiter net for $N \rtimes_{\tau} H$. We present two methods for doing so.

Throughout this section, $N$ and $H$ will be amenable locally compact groups and $\tau$ a continuous group homomorphism from $H$ to $\operatorname{Aut}(N)$. Also, $\left(f_{\alpha}\right)_{\alpha}$ and $\left(g_{\beta}\right)_{\beta}$ will be Reiter nets in $L^{1}(N)_{1}^{+}$and $L^{1}(H)_{1}^{+}$, respectively.

Theorem 3.1 (Method 1). Let $E_{\alpha, \beta}:=f_{\alpha} \times g_{\beta} \delta^{-1}$. The net $\left(E_{\alpha, \beta}\right)_{\alpha, \beta}$ is a Reiter net for $N \rtimes_{\tau} H$ if and only if the following condition holds:

$$
\left\|T_{y} f_{\alpha}-f_{\alpha}\right\|_{L^{1}(N)} \rightarrow 0
$$

uniformly in $y$ on compact subsets of $H$.

Proof. We begin by assuming that (3.1) holds. Then:

$$
\begin{aligned}
& \| l_{(x, y)}^{*} E_{\alpha, \beta}- E_{\alpha, \beta} \|_{L^{1}\left(N \rtimes_{\tau} H\right)} \\
&=\iint\left|l_{x}^{*} T_{y} f_{\alpha}(n) l_{y}^{*} g_{\beta}(h)-f_{\alpha}(n) g_{\beta}(h)\right| d \lambda_{H}(h) d \lambda_{N}(n) \\
& \leq\left\|\left(l_{x}^{*} T_{y} f_{\alpha}\right)-f_{\alpha}\right\|_{L^{1}(N)}\left\|l_{y}^{*} g_{\beta}\right\|_{L^{1}(H)} \\
& \quad+\left\|f_{\alpha}\right\|_{L^{1}(N)}\left\|l_{y}^{*} g_{\beta}-g_{\beta}\right\|_{L^{1}(H)} \\
& \leq\left\|l_{x}^{*}\left(T_{y} f_{\alpha}-f_{\alpha}\right)\right\|_{L^{1}(N)}+\left\|l_{x}^{*} f_{\alpha}-f_{\alpha}\right\|_{L^{1}(N)} \\
& \quad+\left\|l_{y}^{*} g_{\beta}-g_{\beta}\right\|_{L^{1}(H)} .
\end{aligned}
$$

Each of these terms tends to zero uniformly on compact subsets of $N \rtimes_{\tau} H$, so $\left(E_{\alpha, \beta}\right)_{\alpha, \beta}$ is a Reiter net. 
For the converse we will apply similar triangle inequalities to get, for $y \in H$ :

$$
\begin{aligned}
\left\|T_{y} f_{\alpha}-f_{\alpha}\right\|_{L^{1}(N)}= & \int_{N} \int_{H}\left|T_{y} f_{\alpha}(n) g_{\beta}(h)-f_{\alpha}(n) g_{\beta}(h)\right| d \lambda_{H}(h) d \lambda_{N}(n) \\
\leq \| & T_{y} f_{\alpha}\left\|_{L^{1}(N)}\right\| g_{\beta}-l_{y}^{*} g_{\beta} \|_{L^{1}(H)} \\
& +\left\|l_{\left(e_{N}, y\right)}^{*} E_{\alpha, \beta}-E_{\alpha, \beta}\right\|_{L^{1}\left(N \rtimes_{\tau} H\right)} .
\end{aligned}
$$

Since each of the terms on the right tends to zero uniformly in $y$ on compact subsets of $H$, so does the left side of the inequality.

Proposition 3.2. There exists a Reiter net $\left(f_{\alpha}\right)_{\alpha} \subset L^{1}(N)_{1}^{+}$that satisfies (3.1).

Proof. Since $N$ and $H$ are amenable, so is the semidirect product $N \rtimes_{\tau} H$. Therefore there is a Reiter net $\left(F_{\alpha}\right)_{\alpha} \subset L^{1}\left(N \rtimes_{\tau} H\right)_{1}^{+}$.

For each $\alpha$, define $f_{\alpha} \in L^{1}(N)$ via

$$
f_{\alpha}(n):=\int_{h \in H} F_{\alpha}(n, h) \delta(h) d \lambda_{H}(h) .
$$

Then it is easily verified that $\left(f_{\alpha}\right)_{\alpha}$ is a net in $L^{1}(N)_{1}^{+}$. Furthermore, for $y \in H$,

$$
\begin{aligned}
\left\|T_{y} f_{\alpha}-f_{\alpha}\right\|_{L^{1}(N)}= & \int_{n \in N} \mid \int_{h \in H} F_{\alpha}\left(\tau_{y^{-1}}(n), h\right) \delta(y) \delta(h) d \lambda_{H}(h) \\
& -\int_{h \in H} F_{\alpha}(n, h) \delta(h) d \lambda_{H}(h) \mid d \lambda_{N}(n) \\
\leq & \int_{n \in N} \int_{h \in H} \mid F_{\alpha}\left(\tau_{y^{-1}}(n), y^{-1} h\right) \delta(y) \delta\left(y^{-1} h\right) \\
& -F_{\alpha}(n, h) \delta(h) \mid d \lambda_{H}(h) d \lambda_{N}(n) \\
= & \int_{N \rtimes_{\tau} H}\left|F_{\alpha}\left(\tau_{y^{-1}}(n), y^{-1} h\right)-F_{\alpha}(n, h)\right| d \lambda_{N \rtimes_{\tau} H}(n, h) \\
= & \left\|l_{\left(e_{N}, y\right)}^{*} F_{\alpha}-F_{\alpha}\right\|_{L^{1}\left(N \rtimes_{\tau} H\right)} .
\end{aligned}
$$

Since $\left(F_{\alpha}\right)_{\alpha}$ is a Reiter net, $\left\|l_{\left(e_{N}, y\right)}^{*} F_{\alpha}-F_{\alpha}\right\|_{L^{1}\left(N \rtimes_{\tau} H\right)} \rightarrow 0$ uniformly in $y$ on compact subsets of $H$. It remains to show that $\left(f_{\alpha}\right)_{\alpha}$ is a Reiter net for $N$. For $x \in N$,

$$
\begin{aligned}
\left\|l_{x}^{*} f_{\alpha}-f_{\alpha}\right\|_{L^{1}(N)} & =\int_{n \in N}\left|\int_{h \in H} F_{\alpha}\left(x^{-1} n, h\right) \delta(h)-F_{\alpha}(n, h) \delta(h) d \lambda_{H}(h)\right| d \lambda_{N}(n) \\
& \leq \int_{n \in N} \int_{h \in H}\left|F_{\alpha}\left(x^{-1} n, h\right)-F_{\alpha}(n, h)\right| \delta(h) d \lambda_{H}(h) d \lambda_{N}(n) \\
& =\left\|l_{\left(x, e_{H}\right)}^{*} F_{\alpha}-F_{\alpha}\right\|_{L^{1}\left(N \rtimes_{\tau} H\right) .}
\end{aligned}
$$

Again, since $\left(F_{\alpha}\right)_{\alpha}$ is a Reiter net, so is $\left(f_{\alpha}\right)_{\alpha}$.

Remark 3.3. Janzen 8 provides a similar result for the existence of a Følner net which satisfies a condition analogous to (3.1) in the case where $\delta$ is constantly one.

Remark 3.4. If $\left(E_{\alpha, \beta}\right)_{\alpha, \beta}$ is a Reiter net, then so is any subnet. In particular, if $\left(f_{k}\right)_{k=1}^{\infty}$ and $\left(g_{k}\right)_{k=1}^{\infty}$ are Reiter sequences for $N$ and $H$ respectively, which satisfy the conditions of the theorem, then the diagonal sequence $\left(f_{k} \times g_{k} \delta^{-1}\right)_{k=1}^{\infty}$ is a Reiter sequence for $N \rtimes_{\tau} H$. 
Theorem 3.5 (Method 2). For $n \in N, h \in H$, let $D_{\alpha, \beta} \in L^{1}\left(N \rtimes_{\tau} H\right)$ be given by $D_{\alpha, \beta}(n, h):=T_{h} f_{\alpha}(n) g_{\beta}(h) \delta\left(h^{-1}\right)$. If each $g_{\beta}$ is compactly supported, then there exists a subnet of $\left(D_{\alpha, \beta}\right)_{\alpha, \beta}$ which is a Reiter net for $N \rtimes_{\tau} H$.

Proof. Observe that

$$
\begin{aligned}
\| l_{(x, y)}^{*} D_{\alpha, \beta}- & D_{\alpha, \beta} \|_{L^{1}\left(N \rtimes_{\tau} H\right)} \\
= & \int\left|l_{x}^{*} T_{h} f_{\alpha}(n) l_{y}^{*} g_{\beta}(h)-T_{h} f_{\alpha}(n) g_{\beta}(h)\right| \delta\left(h^{-1}\right) d \lambda_{G}(n, h) \\
= & \iint\left|T_{h} l_{\tau_{h^{-1}}(x)}^{*} f_{\alpha}(n) l_{y}^{*} g(h)-T_{h} f_{\alpha}(n) g_{\beta}(h)\right| d \lambda_{N}(n) d \lambda_{H}(h) \\
\leq & \iint\left|T_{h}\left(l_{\tau_{h^{-1}}(x)}^{*} f_{\alpha}-f_{\alpha}\right)(n) \| l_{y}^{*} g_{\beta}(h)\right| d \lambda_{N}(n) d \lambda_{H}(h) \\
& \quad+\iint\left|T_{h} f_{\alpha}(n)\right|\left|l_{y}^{*} g_{\beta}(h)-g_{\beta}(h)\right| d \lambda_{N}(n) d \lambda_{H}(h) \\
= & \int\left\|l_{\tau_{(y h)^{-1}(x)}^{*}}^{*} f_{\alpha}-f_{\alpha}\right\|_{L^{1}(N)}\left|g_{\beta}(h)\right| d \lambda_{H}(h)+\left\|l_{y}^{*} g_{\beta}-g_{\beta}\right\| .
\end{aligned}
$$

For $K \subset N \rtimes_{\tau} H$ compact and $\varepsilon>0$ there exists $\beta_{K, \varepsilon}$ such that for $\beta \geq \beta_{K, \varepsilon}$,

$$
\left\|l_{y}^{*} g_{\beta}-g_{\beta}\right\|_{L^{1}(H)}<\varepsilon / 2 \quad \forall y \in K_{H}=\{y:(x, y) \in K \text { for some } x \in N\} .
$$

For each such $\beta$ there is an $\alpha_{\beta, K, \varepsilon}$ such that for $\alpha \geq \alpha_{\beta, K, \varepsilon}$ for all $h \in \operatorname{supp}\left(g_{\beta}\right)$ and all $(x, y) \in K$,

$$
\left\|l_{\tau_{(y h)^{-1}(x)}^{*}}^{*} f_{\alpha}-f_{\alpha}\right\|_{L^{1}(N)}<\varepsilon / 2 .
$$

Consider the directed set $\Lambda$, where each element of $\Lambda$ is a quadruple consisting of a compact subset $K \subset G$, an $\varepsilon>0$, a $\beta \geq \beta_{K, \varepsilon}$, and an $\alpha \geq \alpha_{\beta, K, \varepsilon}$. The order we put on $\Lambda$ is $\preceq$, where $\left(K_{1}, \varepsilon_{1}, \beta_{1}, \alpha_{1}\right) \preceq\left(K_{2}, \varepsilon_{2}, \beta_{2}, \alpha_{2}\right)$ if $K_{1} \subset K_{2}, \varepsilon_{1} \geq \varepsilon_{2}$, $\beta_{1} \leq \beta_{2}$, and $\alpha_{1} \leq \alpha_{2}$. From the above observations, it is apparent that for any $\varepsilon$ and $K$, there exists $\alpha$ and $\beta$ such that $\left\|l_{(x, y)}^{*} D_{\alpha, \beta}-D_{\alpha, \beta}\right\|<\varepsilon$ for all $(x, y) \in K$.

Then $\left(E_{\alpha, \beta}\right)_{K, \varepsilon, \beta, \alpha}$ is a subnet of $\left(E_{\alpha, \beta}\right)_{\alpha, \beta}$ which is a Reiter net for $N \rtimes_{\tau} H$.

Remark 3.6. If $N \rtimes_{\tau} H$ is $\sigma$-compact, then we can find a sequence of compact sets $\left(F_{n}\right)_{n}$ such that $F_{n} \subset F_{n+1}$ and $N \rtimes_{\tau} H=\bigcup F_{n}$. In this case, we can find a sequence of elements of $\Lambda$ such that $\left(E_{\alpha_{n}, \beta_{n}}\right)_{n=1}^{\infty}$ is a Reiter sequence.

Example 3.7 ('ax+b' Group). Let $G=\mathbb{R} \rtimes_{\tau} \mathbb{R}^{+}$where $\tau_{a}(b)=a b$ for $a \in \mathbb{R}^{+}$ and $b \in \mathbb{R}$. Let $f_{n}=\frac{1}{2 n} \chi_{[-n, n]}$ and $g_{n}=\frac{1}{2 \ln (n)} \chi_{\left[\frac{1}{n}, n\right]}$. Using the method of Theorem 3.5. $D_{n, m}=\frac{1}{4 n \ln (n)} \chi_{\left\{(a b, a) \mid b \in[-n, n], a \in\left[\frac{1}{m}, m\right]\right\}}$. It follows that $\left(D_{n, n}\right)_{n}$ is a Reiter sequence for $G$. Further details of this example can be found in [16, Example 0.5] or [4].

By applying the method of Proposition 3.2 , we get the Reiter sequence for $N$, $\left(h_{n}\right)_{n}$ given by:

$$
h_{n}(b)= \begin{cases}\frac{n^{2}-1}{4 n^{2} \ln (n)} & \text { if }|b|<1, \\ \frac{n^{2}-|b|}{4 n^{2}|b| \ln (n)} & \text { if } 1 \leq|b|<n^{2}, \\ 0 & \text { if } n^{2} \leq|b| .\end{cases}
$$

Combining this with the sequence $\left(g_{n}\right)_{n}$ using Method 1 gives a Reiter sequence for the ' $a x+b$ ' group which is different from the standard example found in the literature:

$$
E_{n}(b, a)=h_{n}(b) g_{n}(a) a .
$$




\section{FøLNER NETS}

As in Example 3.7 it is often possible to have Reiter nets $\left(f_{\alpha}\right)_{\alpha}$ of the form $f_{\alpha}=\frac{\chi_{A_{\alpha}}}{\lambda\left(A_{\alpha}\right)}$. The sets $\left(A_{\alpha}\right)_{\alpha}$ form a Følner net, so called because of the condition introduced in [3]. Namioka investigated numerous Følner type conditions in [15]. For further reference, the reader is directed to [4, Section 3.6] and [16, Chapter 4].

Definition 4.1. Let $G$ be a locally compact group. A net [resp. sequence] $\left(A_{\alpha}\right)_{\alpha}$ of measurable subsets of $G$ such that $0<\lambda_{G}\left(A_{\alpha}\right)<\infty$ is called a Følner net [resp. Følner sequence] if for any $\varepsilon>0$, and any compact $F \subset G$, there exists $\beta$ such that, for $\alpha>\beta$,

$$
\frac{\lambda_{G}\left(x A_{\alpha} \triangle A_{\alpha}\right)}{\lambda_{G}\left(A_{\alpha}\right)}<\varepsilon \quad \forall x \in F .
$$

Remark 4.2. A net $\left(A_{\alpha}\right)_{\alpha}$ of measurable subsets of $G$ with $0<\lambda_{G}\left(A_{\alpha}\right)<\infty$ is a Følner net if and only if for any $\varepsilon>0$, and any compact $F \subset G$, there exists $\beta$ such that, for $\alpha>\beta$,

$$
\frac{\lambda_{G}\left(x A_{\alpha} \backslash A_{\alpha}\right)}{\lambda_{G}\left(A_{\alpha}\right)}<\varepsilon \quad \forall x \in F .
$$

This is because $\lambda_{G}(A \backslash x A)=\lambda_{G}\left(x^{-1} A \backslash A\right)$ since $\lambda_{G}$ is a left Haar measure.

Remark 4.3. Suppose that $\left(A_{\alpha}\right)_{\alpha}$ is a Følner net for $G$. For each $\alpha$, let $f_{\alpha}(x):=$ $\frac{1}{\lambda_{G}\left(A_{\alpha}\right)} \chi_{A_{\alpha}}(x)$. Then $\left(f_{\alpha}\right)_{\alpha}$ is a Reiter net in $L^{1}(G)_{1}^{+}$.

Janzen [8] studied Følner nets in semidirect products where $\delta$ is constantly 1 . As a consequence of the previous section, we now present one of Janzen's results as a corollary to Theorem 3.1 .

Corollary 4.4 (Janzen). Let $N \rtimes_{\tau} H$ be a semidirect product of locally compact groups such that $\delta \equiv 1$. Suppose $\left(A_{\alpha}\right)_{\alpha},\left(B_{\beta}\right)_{\beta}$ are Følner nets for $N$ and $H$ respectively. Then $\left(A_{\alpha} \times B_{\beta}\right)_{\alpha, \beta}$ is a Følner net for $N \rtimes_{\tau} H$ if and only if

$$
\frac{\lambda_{N}\left(\tau_{y}\left(A_{\alpha}\right) \triangle A_{\alpha}\right)}{\lambda_{N}\left(A_{\alpha}\right)} \rightarrow 0
$$

uniformly in $y$ on compact subsets of $H$.

Proof. Let $f_{\alpha}=\frac{\chi_{A_{\alpha}}}{\lambda_{N}\left(A_{\alpha}\right)}$ and let $g_{\beta}=\frac{\chi_{B_{\beta}}}{\lambda_{H}\left(B_{\beta}\right)}$. By Theorem 3.1. $\left(f_{\alpha} \times g_{\beta}\right)_{\alpha, \beta}$ is a Reiter net if and only if $\left\|T_{y} f_{\alpha}-f_{\alpha}\right\| \rightarrow 0$. But $f_{\alpha} \times g_{\beta}=\frac{\chi_{A_{\alpha} \times B_{\beta}}}{\lambda_{N \rtimes_{\tau} H}\left(A_{\alpha} \times B_{\beta}\right)}$ and $\left\|T_{y} f_{\alpha}-f_{\alpha}\right\|=\frac{\lambda_{N}\left(\tau_{y}\left(A_{\alpha}\right) \triangle A_{\alpha}\right)}{\lambda_{N}\left(A_{\alpha}\right)}$.

We cannot use Theorem 3.1 to generalize this result to the case where $\delta$ is not constantly 1 since in this case $\chi_{A} \times \chi_{B} \delta^{-1}$ is not equal to $\chi_{A \times B}$. In fact, we will now show that if the product of two Følner nets is a Følner net for the semidirect product, then $\delta$ must be constantly 1 .

Lemma 4.5. Let $G=N \rtimes_{\tau} H$, for $N$ and $H$ locally compact amenable groups. Let $\left(A_{\alpha}\right)_{\alpha}$ be a Følner net for $N$ and $\left(B_{\beta}\right)_{\beta}$ be a Følner net for $H$. If $\left(A_{\alpha} \times B_{\beta}\right)_{\alpha, \beta}$ is a Følner net for $G$, then

$$
\frac{\lambda_{N}\left(\tau_{y}\left(A_{\alpha}\right) \backslash A_{\alpha}\right)}{\lambda_{N}\left(A_{\alpha}\right)} \rightarrow 0
$$

uniformly in y on compact sets of $H$. 
Proof. Observe that for $x \in N, y \in H$ we have that

$$
\left(x \tau_{y}\left(A_{\alpha}\right) \backslash A_{\alpha}\right) \times y B_{\beta} \subset\left((x, y) *\left(A_{\alpha} \times B_{\beta}\right)\right) \backslash\left(A_{\alpha} \times B_{\beta}\right) .
$$

Now, since $\left(A_{\alpha} \times B_{\beta}\right)_{\alpha, \beta}$ is a Følner net for $G$,

$$
\frac{\lambda_{G}\left(\left((x, y) *\left(A_{\alpha} \times B_{\beta}\right)\right) \backslash\left(A_{\alpha} \times B_{\beta}\right)\right)}{\lambda_{G}\left(A_{\alpha} \times B_{\beta}\right)} \rightarrow 0
$$

uniformly in $(x, y)$ on compact subsets of $G$. Therefore

$$
\frac{\lambda_{G}\left(\left(x \tau_{y}\left(A_{\alpha}\right) \backslash A_{\alpha}\right) \times y B_{\beta}\right)}{\lambda_{G}\left(A_{\alpha} \times B_{\beta}\right)} \rightarrow 0
$$

uniformly in $(x, y)$ on compact subsets of $G$. Observe that

$$
\begin{aligned}
\frac{\lambda_{G}\left(\left(x \tau_{y}\left(A_{\alpha}\right) \backslash A_{\alpha}\right) \times y B_{\beta}\right)}{\lambda_{G}\left(A_{\alpha} \times B_{\beta}\right)} & =\frac{\lambda_{N}\left(x \tau_{y}\left(A_{\alpha}\right) \backslash A_{\alpha}\right)}{\lambda_{N}\left(A_{\alpha}\right)} \frac{\int_{y B_{\beta}} \delta(h) d \lambda_{H}(h)}{\int_{B_{\beta}} \delta(h) d \lambda_{H}(h)} \\
& =\frac{\lambda_{N}\left(x \tau_{y}\left(A_{\alpha}\right) \backslash A_{\alpha}\right)}{\lambda_{N}\left(A_{\alpha}\right)} \frac{\int_{B_{\beta}} \delta(y) \delta(h) d \lambda_{H}(h)}{\int_{B_{\beta}} \delta(h) d \lambda_{H}(h)} \\
& =\frac{\lambda_{N}\left(x \tau_{y}\left(A_{\alpha}\right) \backslash A_{\alpha}\right)}{\lambda_{N}\left(A_{\alpha}\right)} \delta(y) .
\end{aligned}
$$

Choose a compact $F \subset H$. The set $\left\{e_{N}\right\} \times F$ is compact in $G$, so

$$
\frac{\lambda_{N}\left(\tau_{y}\left(A_{\alpha}\right) \backslash A_{\alpha}\right)}{\lambda_{N}\left(A_{\alpha}\right)} \delta(y) \rightarrow 0
$$

uniformly on $F$. Then since $\delta$ is continuous, it is bounded below on $F$ by some positive value. Therefore

$$
\frac{\lambda_{N}\left(\tau_{y}\left(A_{\alpha}\right) \backslash A_{\alpha}\right)}{\lambda_{N}\left(A_{\alpha}\right)} \rightarrow 0
$$

uniformly on $F$.

Theorem 4.6. If $\left(A_{\alpha} \times B_{\beta}\right)_{\alpha, \beta}$ is a Følner net for $G$, then $\delta \equiv 1$.

Proof. Observe that for $y \in H$ :

$$
\begin{aligned}
\lambda_{N}\left(\tau_{y}\left(A_{\alpha}\right) \backslash A_{\alpha}\right) & \geq \lambda_{N}\left(\tau_{y}\left(A_{\alpha}\right)\right)-\lambda_{N}\left(A_{\alpha}\right) \\
& =\lambda_{N}\left(A_{\alpha}\right)\left(\delta\left(y^{-1}\right)-1\right) .
\end{aligned}
$$

By the lemma, we know that $\frac{\lambda_{N}\left(\tau_{y}\left(A_{\alpha}\right) \backslash A_{\alpha}\right)}{\lambda_{N}\left(A_{\alpha}\right)} \rightarrow 0$ for each $y \in H$. If there exists a $y_{0} \in H$ such that $\delta\left(y_{0}^{-1}\right)>1$, then for all $\alpha, \frac{\lambda_{N}\left(\tau_{y_{0}}\left(A_{\alpha}\right) \backslash A_{\alpha}\right)}{\lambda_{N}\left(A_{\alpha}\right)}>\delta\left(y_{0}^{-1}\right)-1$, which is a contradiction, so $\delta \equiv 1$.

As indicated by Example 3.7. the method of Theorem 3.5 does apply, even if $\delta$ is not constantly 1 . The following corollary to Theorem 3.5 was proven directly as Theorem 2.3.8 in the author's thesis [21].

Corollary 4.7. Let $N \rtimes_{\tau} H$ be the semidirect product of two locally compact groups. Suppose that $\left(A_{\alpha}\right)_{\alpha}$ and $\left(B_{\beta}\right)_{\beta}$ are Følner nets for $N$ and $H$ respectively such that each $B_{\beta}$ is compact. Then there is a subnet of $\left(\left\{\left(\tau_{h}(n), h\right): n \in A_{\alpha}, h \in B_{\beta}\right\}\right)_{\alpha, \beta}$ which is a Følner net for $N \rtimes_{\tau} H$. 
Proof. It suffices to observe that for $n \in N, h \in H, A \subset N$, and $B \subset H$,

$$
T_{h} \chi_{A}(n) \chi_{B}(h) \delta\left(h^{-1}\right)=\chi_{\left\{\left(\tau_{y}(x), y\right): x \in A, y \in B\right\}}
$$

and

$$
\lambda_{N \rtimes_{\tau} H}\left(\left\{\left(\tau_{y}(x), y\right): x \in A, y \in B\right\}\right)=\lambda_{N}(A) \lambda_{H}(B) .
$$

\section{Semigroups}

Results analogous to those presented in previous sections can be formulated for semigroups. This section will deal with discrete semigroups. Rather than using a left Haar measure, we consider the counting measure, and the concept of a semidirect product is suitably modified. It is well-known that a semigroup $S$ is left amenable if and only if there is a net $\left(f_{\alpha}\right)_{\alpha}$ of elements in $\ell^{1}(S)_{1}^{+}$for which $\left\|l_{x}^{*} f_{\alpha}-f_{\alpha}\right\| \rightarrow 0$ for all $x \in S$ ([2], [15]). Since $S$ has the discrete topology, the collection of such nets is precisely the Reiter nets for $S$.

Definition 5.1. Let $U$ and $V$ be semigroups. Assume that $V$ acts on $U$ on the left; i.e., assume that there is a semigroup homomorphism $\tau$ from $V$ to $\operatorname{End}(U)$ such that for each $v \in V$ there exists $\tau_{v}: U \rightarrow U$ such that $\tau_{v_{1}}\left(\tau_{v_{2}}(u)\right)=\tau_{v_{1} v_{2}}(u)$ for all $u \in U, v_{1}, v_{2} \in V$.

We say that $S=U \rtimes_{\tau} V$ is the semidirect product of $U$ and $V$ with respect to $\tau$ if $S$ is the semigroup consisting of elements of the form $(u, v)$, where $u \in U$ and $v \in V$ equipped with multiplication given by

$$
\left(u_{1}, v_{1}\right)\left(u_{2}, v_{2}\right)=\left(u_{1} \tau_{v_{1}}\left(u_{2}\right), v_{1} v_{2}\right) .
$$

Using $\tau$, we can define a right action of $V$ on $\ell^{\infty}(U)$ by

$$
\begin{gathered}
T_{v}: \ell^{\infty}(U) \rightarrow \ell^{\infty}(U) \\
\phi \mapsto T_{v} \phi,
\end{gathered}
$$

where $\left(T_{v} \phi\right)(u)=\phi\left(\tau_{v}(u)\right)$. So we get a left action on $\ell^{\infty}(U)^{*}$ by considering $T_{v}^{*}$ for each $v \in V$. Unlike in the group case, each $T_{v}^{*}$ is not necessarily isometric, but $\left\|T_{v}^{*}\right\| \leq 1$. If $U=V$ and $\tau_{v}(u)=v u$, then we denote $T_{v}$ by $l_{v}$.

We say that a net, $\left(f_{\alpha}\right)_{\alpha}$, in $\ell^{1}(U)_{1}^{+}$is a Reiter net if

$$
\left\|l_{u}^{*} f_{\alpha}-f_{\alpha}\right\|_{\ell^{1}(U)} \rightarrow 0
$$

uniformly in $u$ on compact subsets of $U$.

Remark 5.2. Since $U$ has the discrete topology, the uniform convergence on compact (i.e. finite) subsets of $U$ is equivalent to convergence for all $u \in U$. This makes our definition of Reiter nets equivalent to that of strongly regular nets found in [11, [12, and [13].

Left amenability of a semigroup $U$ is defined in terms of a left-invariant mean (see 2]). For simplicity, we remark that $U$ is left amenable if and only if there is a Reiter net in $\ell^{1}(U)_{1}^{+}$. The counting measure on the semidirect product is precisely the product of the counting measures on $N$ and $H$. For the remainder of this section, $U$ and $V$ will be semigroups and $\tau$ a semigroup homomorphism from $V$ to $\operatorname{End}(U)$. As well, $\left(f_{\alpha}\right)_{\alpha}$ and $\left(g_{\beta}\right)_{\beta}$ will be Reiter nets for $U$ and $V$ respectively.

Theorem 5.3. Assume that $\left\|T_{y}^{*} f_{\alpha}-f_{\alpha}\right\| \rightarrow 0$ uniformly in $y$ on compact subsets of $V$. Then $\left(E_{\alpha, \beta}\right)_{\alpha, \beta}$ is a Reiter net for $U \rtimes_{\tau} V$, where $E_{\alpha, \beta}(u, v):=f_{\alpha}(u) g_{\beta}(v)$. 
Proof. The proof proceeds in a similar fashion to that of Theorem 3.1. We safely suppress the details.

Remark 5.4. Unlike in the group case, there is not always a Reiter net which satisfies the condition of the theorem. Indeed, there are examples of semidirect products of left-amenable semigroups which are not left amenable (see [9, 3.6]).

Theorem 5.5. Define $D_{\alpha, \beta} \in \ell^{1}\left(U \rtimes_{\tau} V\right)$ via $D_{\alpha, \beta}(u, v):=T_{v}^{*} f_{\alpha}(u) g_{\beta}(v)$. Assume that each $g_{\beta}$ is finitely supported. Further suppose that for each $v \in V$ the net $\left(T_{v}^{*} f_{\alpha}\right)_{\alpha}$ is a Reiter net. Then there is a subnet of $\left(D_{\alpha, \beta}\right)_{\alpha, \beta}$ which is a Reiter net for $U \rtimes_{\tau} V$.

Proof. It is straightforward to verify that $l_{(x, y)}^{*} D_{\alpha, \beta}(u, v)=l_{x}^{*} T_{v}^{*} f_{\alpha}(u) l_{y}^{*} g_{\beta}(v)$. It is then apparent that

$$
\left\|l_{(x, y)}^{*} D_{\alpha, \beta}-D_{\alpha, \beta}\right\| \leq \sum_{v \in V}\left\|l_{x}^{*} T_{v}^{*} f_{\alpha}-T_{v}^{*} f_{\alpha}\right\| g_{\beta}(v)+\left\|l_{y}^{*} g_{\beta}-g_{\beta}\right\| .
$$

The remainder of this proof mimics that of Theorem 3.5. We again suppress the details.

Remark 5.6. For $x \in U$ and $v \in V, l_{\tau_{v}(x)}^{*} T_{v}^{*} f_{\alpha}=T_{v}^{*} l_{x}^{*} f_{\alpha}$, so if $\tau: V \rightarrow \operatorname{Sur}(U)$, then $U \rtimes_{\tau} V$ is left amenable. This provides a new proof of a result of Klawe 9, $3.4]$.

Remark 5.7. Explicit constructions of Reiter nets for bicyclic semigroups can be found in [13.

\section{REFERENCES}

[1] Ching Chou, On topologically invariant means on a locally compact group, Trans. Amer. Math. Soc. 151 (1970), 443-456. MR0269780 (42:4675)

[2] Mahlon M. Day, Amenable semigroups, Illinois J. Math. 1 (1957), 509-544. MR0092128 $(19: 1067 \mathrm{c})$

[3] Erling Følner, On groups with full Banach mean value, Math. Scand. 3 (1955), 243-254. MR0079220 (18:51f)

[4] Frederick P. Greenleaf, Invariant means on topological groups and their applications, Van Nostrand Mathematical Studies, No. 16, Van Nostrand Reinhold Co., New York, 1969. MR0251549 (40:4776)

[5] Edwin Hewitt and Kenneth A. Ross, Abstract harmonic analysis. Vol. I, second ed., Grundlehren der Mathematischen Wissenschaften [Fundamental Principles of Mathematical Sciences], vol. 115, Structure of topological groups, integration theory, group representations, Springer-Verlag, Berlin, 1979. MR551496 (81k:43001)

[6] Neil Hindman and Dona Strauss, Density and invariant means in left amenable semigroups, Topology and its Applications. In press, corrected proof (2009).

[7] A. Hulanicki, Means and Følner condition on locally compact groups, Studia Math. 27 (1966), 87-104. MR0195982 (33:4178)

[8] David Janzen, Følner nets for semidirect products of amenable groups, Canad. Math. Bull. 51 (2008), no. 1, 60-66. MR.2384739

[9] Maria Klawe, Semidirect product of semigroups in relation to amenability, cancellation properties, and strong Følner conditions, Pacific J. Math. 73 (1977), no. 1, 91-106. MR0470609 $(57: 10357)$

[10] Anthony To-Ming Lau, Uniformly continuous functionals on the Fourier algebra of any locally compact group, Trans. Amer. Math. Soc. 251 (1979), 39-59. MR.531968 (80m:43009)

[11] Anthony To-Ming Lau, Hiromichi Miyake, and Wataru Takahashi, Approximation of fixed points for amenable semigroups of nonexpansive mappings in Banach spaces, Nonlinear Anal. 67 (2007), no. 4, 1211-1225. MR.2325374(2008e:47122) 
[12] Anthony To-Ming Lau, Naoki Shioji, and Wataru Takahashi, Existence of nonexpansive retractions for amenable semigroups of nonexpansive mappings and nonlinear ergodic theorems in Banach spaces, J. Funct. Anal. 161 (1999), no. 1, 62-75. MR.1670206 (99j:47079)

[13] Anthony To-Ming Lau and Yong Zhang, Fixed point properties of semigroups of nonexpansive mappings, J. Funct. Anal. 254 (2008), no. 10, 2534-2554. MR 2406686

[14] Horst Leptin, Sur l'algèbre de Fourier d'un groupe localement compact, C. R. Acad. Sci. Paris Sér. A-B 266 (1968), A1180-A1182. MR0239002 (39:362)

[15] I. Namioka, Følner's conditions for amenable semi-groups, Math. Scand. 15 (1964), 18-28. MR0180832(31:5062)

[16] Alan L. T. Paterson, Amenability, Mathematical Surveys and Monographs, vol. 29, American Mathematical Society, Providence, RI, 1988. MR961261 (90e:43001)

[17] H. Reiter, The convex hull of translates of a function in $L^{1}$, J. London Math. Soc. 35 (1960), 5-16. MR0110763(22:1631)

[18] Zhong-Jin Ruan, The operator amenability of $A(G)$, Amer. J. Math. 117 (1995), no. 6, 1449-1474. MR:1363075 (96m:43001)

[19] Ross Stokke, Approximate diagonals and Følner conditions for amenable group and semigroup algebras, Studia Math. 164 (2004), no. 2, 139-159. MR2079635 (2005f:43002)

[20] _ Q Quasi-central bounded approximate identities in group algebras of locally compact groups, Illinois J. Math. 48 (2004), no. 1, 151-170. MR2048220 (2005g:43005)

[21] Benjamin Willson, Følner conditions and semidirect products related to amenability of semigroups and groups, Master's thesis, University of Alberta, Edmonton, Alberta, Fall 2006.

Department of Mathematical and Statistical Sciences, University of Alberta, Edmonton, Alberta, T6G 2G1, Canada

E-mail address: bwillson@math.ualberta.ca 